\title{
Terapia de Contensáo Induzida Associada à Eletroestimulaçáo Funcional na Paresia de Membro Superior
}

\author{
Constraint Induced Movement Therapy Associated with Functional Electrical Stimulation on Upper Limb Paresis
}

\author{
Flávia Priscila de Paiva Silva', Stefânia Portugal Gonçalves², Sidney \\ Benedito Silva ${ }^{3}$, Denise Fortes Chibeni Ramos Rios ${ }^{4}$, Adriana Teresa Silva ${ }^{5}$
}

\begin{abstract}
RESUMO
Objetivo. Comparar a terapia de contensão induzida (TCI) e esta associada à eletroestimulaçáo funcional (FES) no membro superior parético de indivíduos com acidente vascular cerebral (AVC). Método. Amostra de 12 indivíduos divididos em 2 grupos ( $\mathrm{n}=6$ ): grupo I - TCI e grupo II - TCI associada à FES. Os indivíduos deveriam apresentar histórico de AVC, hemiparesia, no mínimo $10^{\circ}$ de extensão do punho e estado cognitivo preservado. Foram avaliados antes e ao final das sessóes e após 2 meses do término dessas. Para avaliação foram utilizados: Mini-Exame do Estado Mental, Escala de Avaliação Motora de FuglMeyer, goniometria e escala manual de força muscular. Realizou-se 10 sessóes com duração de 3 horas por um período de 2 semanas, sendo que o grupo II realizou 10 minutos a mais para aplicaçáo da FES. Resultados. Obteve-se uma melhora significante na função do membro parético no grupo I; na goniometria da extensão do punho em ambos os grupos e na força muscular dos músculos flexores do punho no grupo II. Comparando-se os grupos, não houve diferença estatística entre eles. Conclusáo. Não houve diferença entre as técnicas utilizadas, devendo-se ressaltar que independente do tipo da técnica utilizada o indivíduo se beneficiará com o tratamento fisioterápico.
\end{abstract}

Unitermos. Acidente Vascular Cerebral, Reabilitação, Estimulação Elétrica.

Citação. Silva FPP, Gonçalves SP, Silva SB, Rios DFCR, Silva AT. Terapia de Contensão Induzida Associada à Eletroestimulação Funcional na Paresia de Membro Superior.

Trabalho realizado na Universidade do Vale do Sapucaí -UNIVÁS, Pouso Alegre-MG, Brasil.

1. Fisioterapeuta, Especialista em Fisioterapia Neurofuncional ISCMSP, São Paulo-SP, Brasil.

2. Fisioterapeuta, Pouso Alegre-MG, Brasil.

3. Fisioterapeuta, docente da Universidade do Vale do Sapucaí, Pouso AlegreMG, Brasil.

4. Fisioterapeuta, docente da Universidade do Vale do Sapucaí, Pouso AlegreMG, Brasil.

5. Fisioterapeuta, mestranda na Neurologia UNICAMP, docente da Universidade do Vale do Sapucaí, Pouso Alegre-MG, Brasil.

\begin{abstract}
Objective. Compare the constraint induced movement therapy (TCI) and it associated with functional electrical stimulation (FES) in upper limb of patients with stroke. Method. The sample included 12 patients divided into 2 groups $(n=6)$ - group I - TCI and group II - TCI associated with FES. Individuals should have a history of stroke, hemiparesis, at least $10^{\circ}$ of wrist extension and preserved cognitive status. Were evaluated before and in the end of the session and after 2 months of completing these. For evaluation were used: MiniMental State Examination, Fugl-Meyer Motor Assessment Scale, goniometry and manual muscle strength scale. We conducted 10 sessions lasting 3 hours for a period of two weeks, while Group II held 10 minutes longer for the application of FES. Results. We found a significant improvement in the paretic limb function in group I, in goniometry of wrist extension in both groups and muscle strength of the wrist flexor muscles in group II. Comparing the groups, there was no statistical difference between them. Conclusion. There was no difference between techniques and should be emphasized that regardless of the type of technique the individual will benefit from physiotherapy treatment.
\end{abstract}

Keywords. Stroke, Rehabilitation, Electrical Stimulation.

Citation. Silva FPP, Gonçalves SP, Silva SB, Rios DFCR, Silva AT. Constraint Induced Movement Therapy Associated with Functional Electrical Stimulation on Upper Limb Paresis.
Endereço para correspondência: Adriana Teresa Silva Av. Pref. Tuany Toledo, 470 CEP 37550-000, Pouso Alegre-MG, Brasil. E-mail: adrianat.silva@yahoo.com.br

Original

Recebido em: 03/02/11

Aceito em: 09/08/11

Conflito de interesses: não 


\section{INTRODUÇÃO}

O Acidente Vascular Cerebral (AVC) define-se como um déficit neurológico focal súbito, resultante da interrupção sanguínea de uma determinada região encefálica, com sintomas de duração maior que 24 horas e/ou presença de lesão cerebral em exame de imagem ${ }^{1}$.

O AVC, atualmente, é uma das principais causas de incapacidades neurológicas em adultos, acometendo a função das extremidades de membros, controle motor, equilíbrio, força e mobilidade².

Os programas terapêuticos para pacientes com AVC mostraram-se capazes de melhorar os resultados funcionais, sendo a fisioterapia um componente indispensável neste processo ${ }^{3}$. Uma intervenção que vem sendo utilizada para o tratamento da extremidade superior parética é a Terapia de Contensão Induzida (TCI) (no inglês: Constraint Induced Moviment Therapy). Esta consiste no treinamento intensivo, prática de repetiçóes funcionais e na contensão do membro superior nãoparético ${ }^{4,5}$.

A eficácia da TCI, que se baseia na superação do uso e desuso, vem sendo documentada em vários estudos e tem demonstrado ser uma opçáo terapêutica para os indivíduos com lesões hemisféricas que desenvolvem hemiparesia ${ }^{5}$.

Outra intervenção terapêutica utilizada na reabilitação após o AVC é a Eletroestimulação Funcional, (FES), (no inglês: Functional Electrical Stimulation), que induz potenciais de ação no nervo motor, provocando ativação de unidades motoras. Esse tipo de modalidade terapêutica vem se mostrando eficaz em indivíduos com membro superior parético. Têm sido relatados: ganho de força muscular da região estimulada, melhora do controle motor voluntário e diminuiçáo da espasticidade ${ }^{6}$.

Ao realizar a revisão de literatura pode-se constatar que não existem estudos científicos comparando as duas técnicas terapêuticas. Entretanto, há um relato de caso ${ }^{7}$ que associou as duas técnicas obtendo melhora funcional do membro superior parético.

Em virtude disso, o presente estudo visou comparar a técnica TCI associada ou não à FES para avaliar os resultados destas, buscando obter a melhor terapêutica no processo de recuperação de indivíduos com hemiparesia de membro superior decorrente de AVC.

\section{MÉTODO}

A amostra do estudo consistiu em 12 indivíduos do ambulatório de Fisioterapia do Hospital das Clínicas Samuel Libânio, localizado no município de Pouso Alegre - MG, que haviam sofrido AVC, com sequela de hemiparesia de membro superior e que apresentassem no mínimo $10^{\circ}$ de extensão de punho. A faixa etária variou entre 30 e 75 anos, e os indivíduos em questão deveriam apresentar um bom estado cognitivo, o qual foi avaliado através do Mini-Exame do Estado Mental (MEEM) ${ }^{8,9}$.

Para o MEEM o ponto de corte é frequentemente ajustado para o nível educacional. Conforme alguns autores já sugeriram, utilizou-se o corte 24 para pessoas com escolaridade acima de 9 anos de estudo, enquanto o corte 17 foi utilizado para aqueles com menor escolaridade?

Foram excluídos do estudo os indivíduos com histórico de trauma cranioencefálico e aqueles que apresentaram alguma contra-indicação para a utilização da FES.

O presente estudo foi previamente analisado e autorizado pelo Comitê de Ética em Pesquisa da Universidade do Vale do Sapucaí - UNIVÁS, sob o protocolo número 997/08. Os indivíduos assinaram o Termo de Consentimento Livre e Esclarecido.

\section{Procedimento}

Os indivíduos foram avaliados no início e ao final do protocolo de tratamento; e também após 2 meses foi aplicado um pós-teste para se verificar a permanência dos resultados obtidos. As avaliaçóes foram realizadas por um mesmo avaliador.

Para avaliação motora funcional dos indivíduos foi utilizada a pontuação específica para membro superior da Escala de Avaliaçâo Motora de Fugl-Meyer (FMA) ${ }^{10}$. Para avaliação da amplitude de movimento (ADM), foi realizada a goniometria ativa da extensão do punho partindo da flexáo com cotovelo fletido e estendido através de um goniômetro universal ${ }^{11}$. Foi avaliada a força muscular dos extensores e flexores do punho através da escala manual de força muscular, utilizando a classificação de 0 a $5^{12}$.

Os indivíduos foram divididos em 2 grupos, selecionados por amostragem aleatória simples $(n=6)$. O grupo I realizou um protocolo adaptado de tratamento que consistiu em 5 sessôes semanais com duração de 3 horas no período de 2 semanas, onde os indivíduos fo- 
ram estimulados a realizar atividades de vida diária com imobilização contralateral ao membro superior parético através de uma tipóia. Já o grupo II realizou o mesmo protocolo associado a 10 minutos de aplicação da FES ao final de cada sessão.

Durante o protocolo de tratamento os indivíduos realizaram tarefas adaptadas que consistiram em 21 exercícios com graus progressivos de dificuldade realizados em pequenas etapas (shaping) ${ }^{13}$, estes eram constituídos por atividades do dia-a-dia, tais como: rosquear recipientes de diferentes tamanhos, pendurar roupas no varal, cortar os alimentos com garfo e faca e escrever utilizando caneta e papel. Todos os sujeitos da pesquisa foram estimulados a utilizar a tipóia em casa durante as atividades de vida diária.

Os indivíduos do grupo II realizaram 10 minutos a mais nas sessões para aplicação da FES nos músculos flexor superficial dos dedos, flexor ulnar do carpo e extensor comum dos dedos, estimulados através de eletrodos posicionados sobre o ponto motor destes, localizados na face anterior (próximo ao epicôndilo medial) e face pósterolateral (próximo ao epicôndilo lateral) visando obter um movimento próximo do funcional.

Para aplicação da FES foi utilizado o aparelho de eletroestimulação Neurodym III da IBRAMED ${ }^{\circledR}$ com número na ANVISA: 10360310012. Este veio aferido da fábrica e foi utilizado somente nesta pesquisa. $\mathrm{O}$ aparelho foi ajustado no programa 7 (FES recíproco). Este possui 2 canais de condução da corrente em forma de onda quadrada bifásica simétrica. Foram usados 3 eletrodos com medida de $5,5 \times 3 \mathrm{~cm}$ e 1 com medida de $5,5 \times 6 \mathrm{~cm}$. A eletroestimulação foi aplicada através dos seguintes parâmetros: tempo on de 5 segundos, tempo off, 10 segundos; tempo de subida da onda de 2 segundos e de descida, 1 segundo, sendo estes valores fixos no aparelho. Foram moduladas a largura do pulso em $275 \mu$ s e a frequência do pulso em $50 \mathrm{~Hz}$. A intensidade da corrente foi ajustada conforme o nível de tolerância de cada paciente ${ }^{14}$.

As sessões foram realizadas em uma mesma sala, com temperatura e ilu- minação adequadas e utilizando-se uma mesa com altura apropriada à realizaçáo das atividades.

\section{Análise Estatística}

Para análise dos resultados foram aplicados os seguintes testes: análise de variância de Friedman e teste de Kruskal-Wallis para comparar os três tempos do estudo (inicial, final e após 2 meses) para cada técnica aplicada e teste de Mann-Whitney para comparar as duas técnicas em cada um dos tempos ${ }^{15}$. Para tanto, utilizou-se o programa SPSS 15.0 e fixou-se em 0,05 ou $5 \%$ o nível de rejeição da hipótese de nulidade.

\section{RESULTADOS}

Dos 12 indivíduos que participaram do estudo, $67 \%$ eram do gênero feminino e $33 \%$, do gênero masculino. Pode-se verificar que $75 \%$ dos AVCs foram diagnosticados em isquêmicos e $25 \%$ em hemorrágicos. O hemicorpo direito foi acometido em $75 \%$ dos indivíduos e em $25 \%$, o esquerdo. A faixa etária variou de 30 a 75 anos $(52,75 \pm 11,87)$.

Ao se avaliar a função motora do membro superior parético nos períodos inicial, final e após 2 meses (Figura $1)$, observou-se que os valores dos períodos final $(109,5)$ e após 2 meses $(108,7)$, no grupo que realizou somente a TCI, foram maiores do que os do período inicial $(94,5)$, $\mathrm{p}=0,009$, evidenciando melhora da função motora.

Em relação a goniometria, tanto na extensão do punho com o cotovelo estendido (Figura 2), quanto com o cotovelo fletido (Figura 3) observou-se, para a técnica
Figura 1 - Média dos resultados obtidos segundo a escala motora de Fugl-Meyer

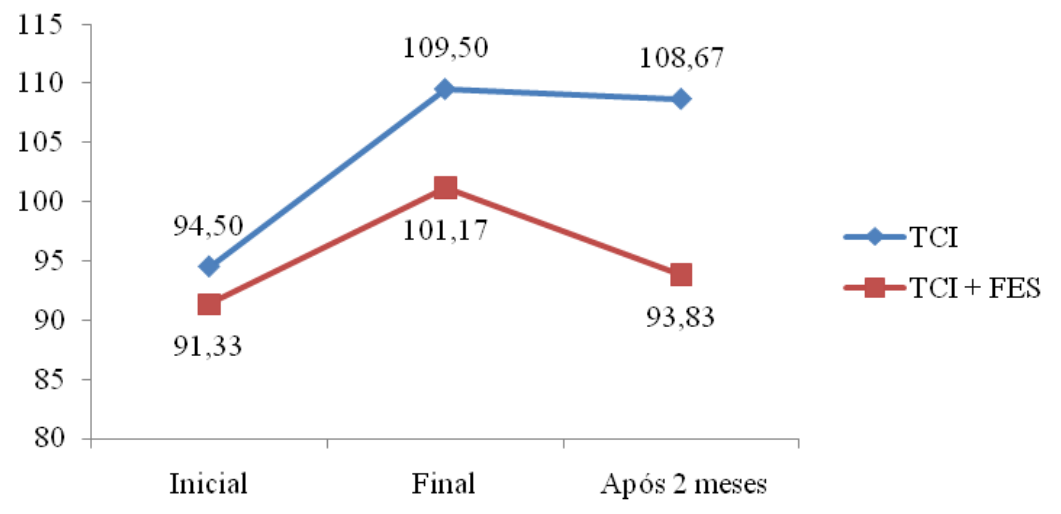

Figura 1. Média dos resultados obtidos segundo a escala motora de Fugl-Meyer. 


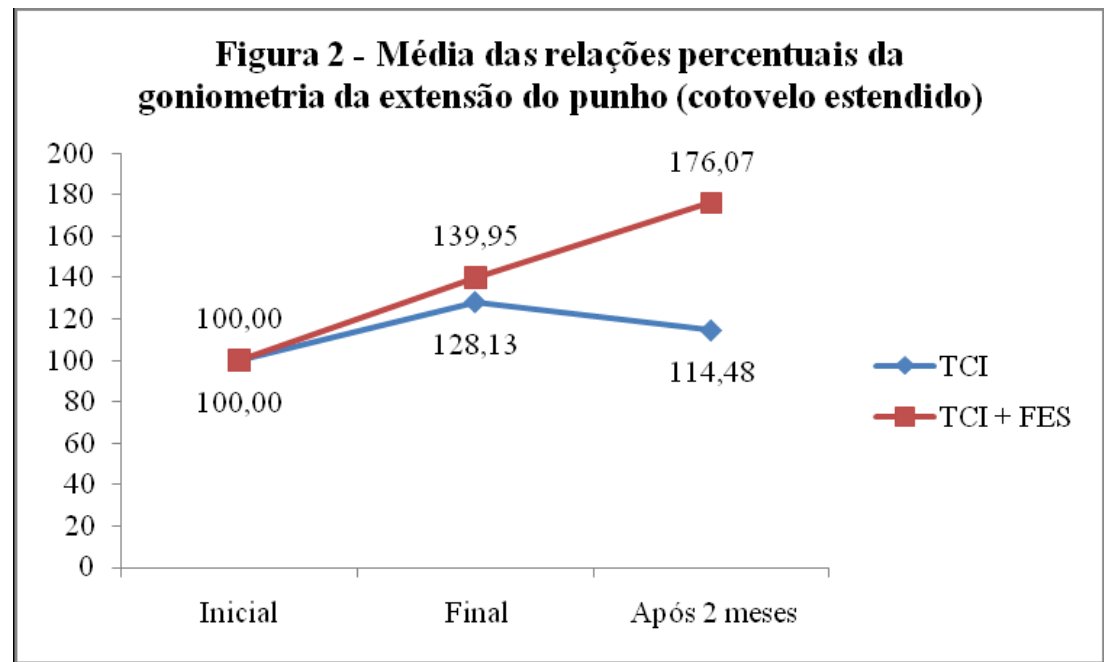

Figura 2. Média das relaçóes percentuais da goniometria da extensão do punho (cotovelo estendido).

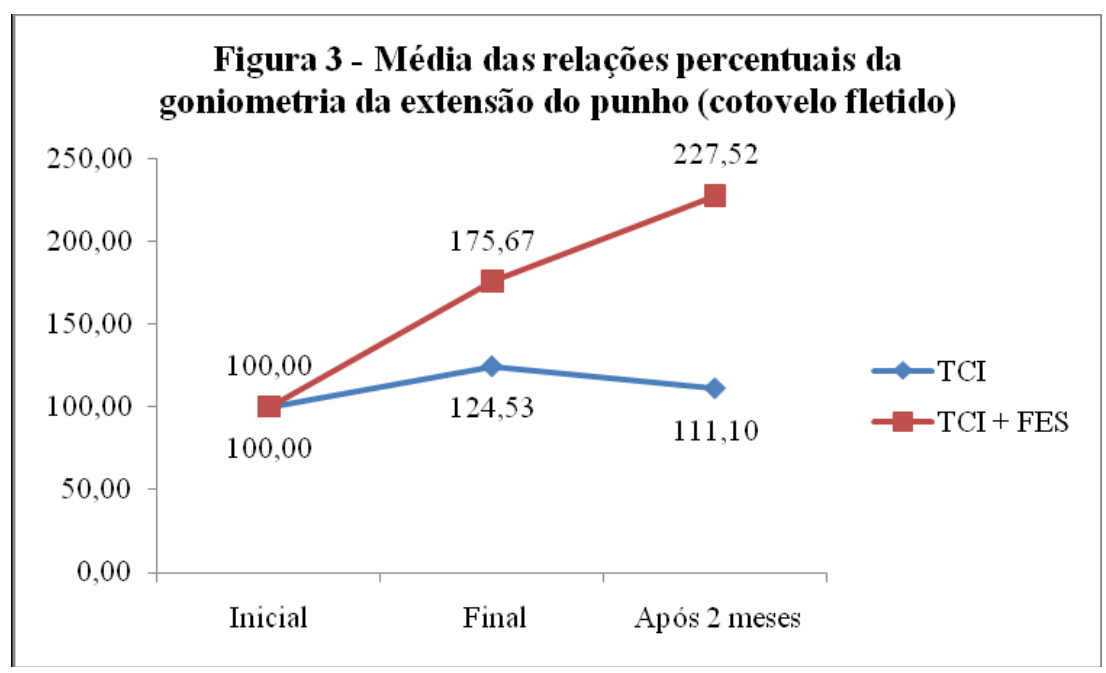

Figura 3. Média das relaçôes percentuais da goniometria da extensão do punho (cotovelo fletido).

TCI, que os valores do período final $\left(128,13^{\circ}\right.$ e $124,53^{\circ}$, respectivamente) foram maiores do que os do período inicial ( $100^{\circ}$ para ambos), com $\mathrm{p}=0,03$ e $\mathrm{p}=0,016$, respectivamente; e para a TCI associada à FES os valores do período após 2 meses $\left(176,07^{\circ}\right.$ e $\left.227,52^{\circ}\right)$ foram maiores do que os do período inicial ( $100^{\circ}$ para ambos), com $\mathrm{p}=0,032$ e $\mathrm{p}=0,03$, respectivamente, evidenciando aumento da ADM.

$\mathrm{Na}$ avaliação da força muscular dos extensores do punho (Figura 4) não houve diferença nos resultados antes e depois do tratamento. Já na avaliação da força muscular dos flexores do punho (Figura 5), no grupo da TCI associada à FES, os resultados se mostraram significativos nos período final $(3,67)$ em relação ao inicial $(2,67)$ e após 2 meses $(3,17)$, com $\mathrm{p}=0,042$.

Ao se comparar os 2 grupos, nos períodos final e após 2 meses do término da terapia, não houve diferença entre os grupos nos critérios avaliados.

\section{DISCUSSÃO}

Neste estudo, obteve-se como resultados uma melhora significante na função do membro parético no grupo I, na goniometria da extensão do punho em ambos os grupos e na força muscular dos músculos flexores do punho no grupo II. Comparando-se os grupos, não houve diferença estatística entre eles.

Ao realizar a revisão de literatura encontrou-se somente um estudo com a TCI e esta associada à FES7. Trata-se do estudo de um caso de AVC crônico submetido à TCI associada à FES durante a realização das atividades propostas pelo protocolo de tratamento. Foram realizadas avaliaçóes inicial, ao final da intervenção e após 10 semanas, sendo realizadas através da escala FMA, Action Research Arm Test, Box and Block, Arm Motor Activity Test e Stroke Impact Scale. O indivíduo foi submetido a 3 horas de terapia diárias, 5 dias na semana por 4 semanas; o tempo de aplicação da FES foi aumentado conforme a tolerância do paciente durante as 4 semanas (1,5 - 2,5 horas). Observou-se uma melhora na função da extremidade superior parética e o paciente relatou uma melhora subjetiva na marcha.

Ao contrário do obtido neste estudo, o estudo descrito anteriormente ${ }^{7}$ encontrou ganhos na função do membro superior parético associando-se TCI e FES, porém a metodologia foi diferente, na qual se aplicou a FES durante as atividades e não 10 minutos a mais após o término da sessão. Alguns pacientes deste estudo, em ambos os grupos, também relataram melhora subjetiva da marcha.

Os dados obtidos ao se avaliar a função da extremidade superior, através da escala FMA, demonstraram 


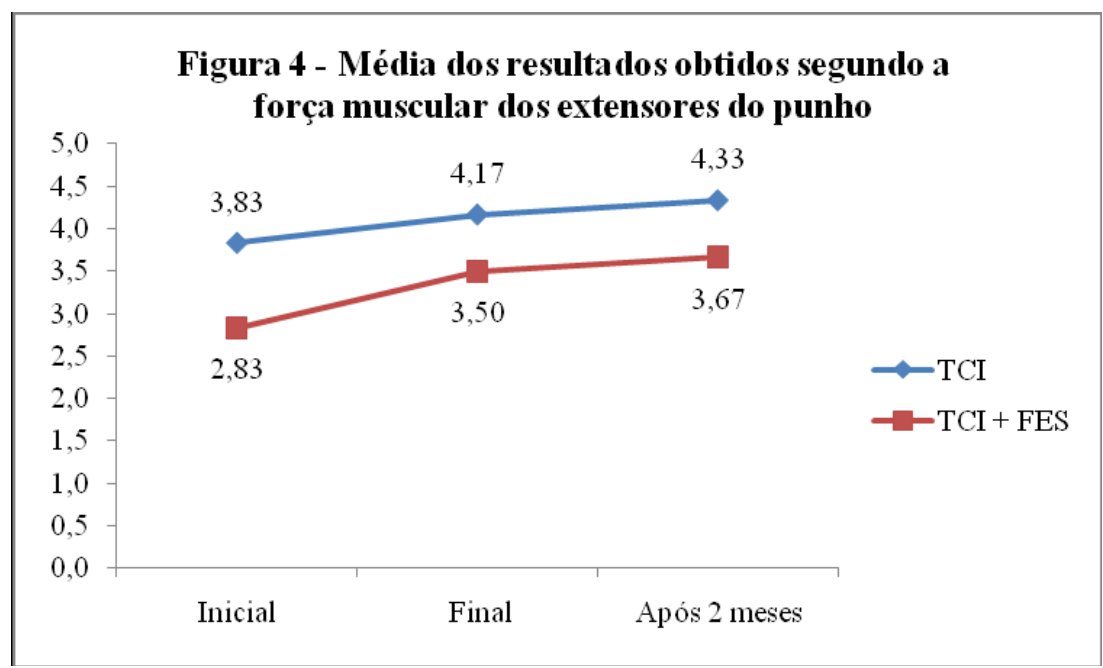

Figura 4. Média dos resultados obtidos segundo a força muscular dos extensores do punho.

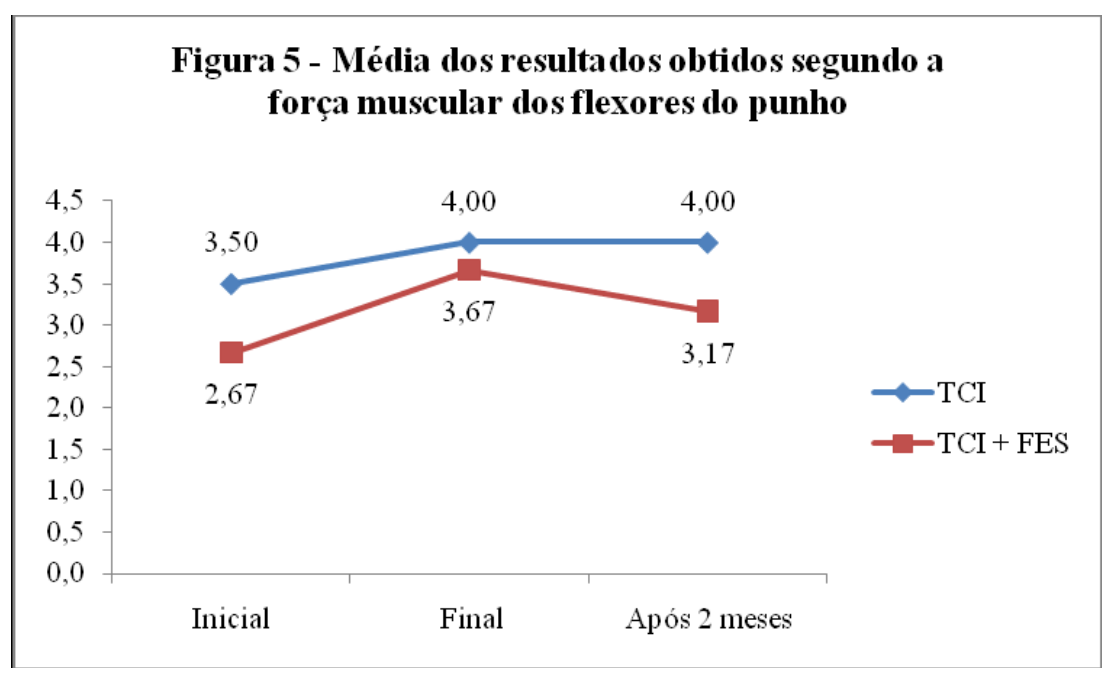

Figura 5. Média dos resultados obtidos segundo a força muscular dos flexores do punho.
$\mathrm{Na}$ revisão de literatura não foram encontrados estudos que avaliaram a força muscular dos extensores e flexores de punho na aplicação do protocolo da TCI e nem desta associada à FES. Contudo, estudo realizado com 60 pacientes hemiparéticos, dividindo-os em um grupo com tratamento padrão e outro com aplicação da FES em extensores de punho ${ }^{24}$, obteve melhora na força isométrica destes, diferente dos resultados obtidos neste estudo.

Os valores atribuídos à força muscular pela escala manual de força são fixos em uma escala numérica que varia de 0 a 5 , o que reduz sua sensibilidade, este fato pode ter influenciado a análise estatística dos resultados, levando a não significância destes na comparação dos grupos.

$\mathrm{O}$ treinamento intensivo e o uso forçado do membro superior parético, realizado na TCI, promovem uma reorganização cortical uso dependente ${ }^{25}$. A reorganizaçâo cortical estimulada pela prática da TCI explica a permanência dos ganhos no grupo da TCI.

Diversos estudos relatam a permanência nos ganhos obtidos na fun- ganhos significantes no grupo que realizou somente a TCI. Em estudos que também realizaram a TCI e utilizaram a FMA para avaliação da função da extremidade superior ${ }^{16-21}$, houve melhora na função, assim como neste estudo.

Em um outro relato de caso pode-se observar ganho na ADM da extensão do punho do sujeito da pesquisa, que foi submetido à TCI, após avaliação final, assim como em neste estudo ${ }^{22}$. Apesar de não haver estudos avaliando a ADM de pacientes submetidos à TCI e à FES, estudos realizados com a FES ${ }^{23,24}$ relataram que esta aplicada aos músculos do punho e dedos promove uma melhora conjunta na ADM e no controle voluntário da contraçáo muscular em pacientes em fase aguda e subaguda do AVC. ção do membro superior em pós-testes em períodos de 2 semanas a 2 anos através da aplicação da TCI, assim como no presente estudo ${ }^{18,19,26-28}$.

Não se encontrou na literatura dados relacionados à permanência de ganhos na ADM de pacientes submetidos à TCI e desta associada à FES. No entanto, pode-se considerar que a permanência dos ganhos na função da extremidade superior poderia promover uma melhora na ADM.

Pode-se observar a permanência dos ganhos obtidos na função motora da extremidade superior parética, em consequência, pode haver melhora da força muscular. Quanto à FES, estudos têm demonstrado recrutamento neuronal em reposta à estimulação elétrica ${ }^{29,30}$, o que poderia estar associado à permanência dos ganhos no grupo da TCI associada à FES. 
Vale-se ressaltar que um paciente do grupo TCI associada à FES entre o período de avaliação final e pósteste (após 2 meses) apresentou quadro clínico de pneumonia, passando por um período de internação. Tal fato pode ter influenciado a piora dos parâmetros avaliativos.

É importante enfatizar que não há na literatura estudos que comparem a TCI e esta associada à FES. O presente estudo apresentou uma amostra pequena que pode ter influenciado nos resultados. Por isso, sugere-se novos estudos com uma amostra maior e com a mesma metodologia.

Além disso, a TCI ou esta associada à FES não apresentou efeitos colaterais perceptíveis, demostrando ser uma técnica segura de tratamento, de baixo custo e fácil aplicabilidade.

\section{CONCLUSÃO}

Pode-se concluir com o presente estudo que não houve diferença estatística entre as técnicas utilizadas, devendo-se ressaltar que independente do tipo da técnica utilizada o indivíduo se beneficiará com o tratamento fisioterapêutico. As técnicas demonstraram ser seguras e com viável aplicação clínica.

\section{REFERÊNCIAS}

1.Barros AF, Fábio SRC, Furkin AM. A rondomized controlled Trial of modified constraint-induced movement therapy for elderly stroke survivors: changes in motor impairment, daily functioning, and quality of life. Arch Phys Med Rahabil 2006;88:273-8.

2.Rodrigues JE, Sá MS, Alouche SR. Perfil dos pacientes acometidos por AVE tratados na clínica escola de fisioterapia da UMESP. Rev Neurocienc 2004;12:117-22.

3.O'Sullivan SB. Acidente vascular encefálico. In: O'Sullivan SB, SCHIMITZ TJ. Fisioterapia: avaliação e tratamento. $2^{\text {a }}$ ed. São Paulo: Manole, 2004, p.519-82.

4.Fritz LS, Light KE, Patterson TS, Behrman AL, Davis SB. Active finger extension predicts outcomes after constraint-induced movement therapy for individuals with hemiparesis after stroke. Stroke 2005;36:1172-77.

http://dx.doi.org/10.1161/01.STR.0000165922.96430.d0

5.Riberto M, Monroy HM, Kaihami HN, Otsubo PPS, Battistella LR. A terapia de restrição como forma de aprimoramento da função de membro superior em pacientes com hemiplegia. Acta Fisiatr 2005;12:15-9.

6.Arantes NF, Vaz DV, Mancini MC, Pereira MSDCI, Pinto FPI, Pinto TPS. Efeitos da estimulação elétrica funcional nos músculos do punho e dedos em indivíduos hemiparéticos: uma revisão sistemática da literatura. Rev Bras Fisioter 2007;11:419-27.
7.Strasser A, Albers B, Schofield K, Dunning K, Levine PG, Hill V et al. FES and forced use therapy for a person with chronic stroke: a case study. J Neurol Phys Ther 2006;30:208.

8.Murden RA, McRae TD, Kaner S, Bucknam ME. Mini-mental state exam scores vary with education in blacks and whites. J Am Geriatr Soc 1991;39:149-55. 9.Brucki SMD, Nitrini R, Caramelli P, Bertolucci PHF, Okamoto IH. Sugestôes para o uso do mini-exame do estado mental no Brasil. Arqui Neuropsiquiatr 2003;61:20-6.

http://dx.doi.org/10.1590/S0004-282X2003000500014

10.Maki T, Quagliato EMAB, Cacho EWA, Paz LPS, Nascimento NH, Inoue MMEA, et al. Estudo de confiabilidade da aplicaçâo da escala de Fugl-Meyer no Brasil. Rev Bras Fisioter 2006;10:177-83.

http://dx.doi.org/10.1590/S1413-35552006000200007

11.Gajdosik RL, Bohannon RW. Clinical measurement of range of motion: review of goniometry emphasizing reliability and validity. Phys Therap 1987;67:1867-72.

12.Kendall FP, McCreary EB., Provance PG. Músculos: Provas e Funçôes. 5a ed. São Paulo: Manole, 2007, 576p.

13.Taub E, Uswatte G, Pidikiti R. Constraint-induced movement therapy: a new family of techniques with broad application to physical rehabilitation - a clinical review. J Rehabil Res Develop 1999; 36:12-6.

14.Santana JM, Filho VJS, Cândido EA, Freire RF. Eletroestimulação functional no controle da espasticidade em indivíduo hemiparético. Rev Fafibe on-line 2005:1-6.

15.Siegel SE, Castellan JR NJ. Estatística não paramétrica para ciências do comportamento. 2a ed. Porto Alegre: Artmed, 2006, 470p.

16.Page SJ, Sueann S, Levine P, Johnson MV, Hughes M. Modified constraint induced therapy: a randomized feasibility and efficacy study. J Rehabil Res Develop; 2001;38:583-90.

17.Bonifer NM, Anderson KM. Application of constraint-induced movement therapy for an individual with severe chronic upper-extremity hemiplegia. Phys Ther; 2003;83:384-98.

18.Grotta JC, Noser EA, Ro T, Boake C, Levin H, Aronowski J, et al. Constraint-induced movement therapy. Stroke 2004;35:2699-701.

http://dx.doi.org/10.1161/01.STR.0000143320.64953.c4

19.Boake C, Noser EA, Ro T, Baraniuk S, Gaber M, Johnson R, et al. Constraint-induced movement therapy during early stroke rehabilitation. Neurorehabil Neural Repair 2007;21:14-24.

http://dx.doi.org/10.1177/1545968306291858

20.Bueno GDP, Lúcio AC, Oberg TD, Cacho EWA. Terapia de restrição e indução modificada do movimento em pacientes hemiparéticos crônicos: um estudo piloto. Fisioter Mov 2008;21:37-44.

21.Souza RD. Terapia de restrição de membro superior não parético e indução de movimento em pacientes hemiparéticos [dissertação]. Campinas: Faculdade de Ciências Medicas da Universidade Estadual de Campinas (UNICAMP); 2008, 84p.

22.Borges MP, Ishiry MN. Aplicação de um protocolo adaptado de duas horas da terapia por contensáo induzida em paciente com sequela de acidente vascular encefálico - estudo de um caso [trabalho de conclusão de curso]. Pouso Alegre: Universidade do Vale do Sapucaí (UNIVÁS); 2008, 76p.

23.Chae J, Bethoux F, Bohinc T, Dobos L, Davis T, Friedl A. Neuromuscular stimulation for upper extremity motor and functional recovery in acute hemiplegia. Stroke 1998;29:975-79.

http://dx.doi.org/10.1161/01.STR.29.5.975

24.Powell J, Pandyan D, Granat M, Cameron M, Stott DJ. Electrical stimulation of wrist extensors in poststroke hemiplegia. Stroke 1999;30:1384-89. http://dx.doi.org/10.1161/01.STR.30.7.1384

25.Assis RD, Massaro AR, Chamlian TR, Silva MF, Ota SM. Terapia de restri- 
ção para uma criança com paralisia cerebral com hemiparesia: estudo de caso. Acta Fisiatr 2007; 14:62-5.

26.Dettmers C, Teske U, Hamzei F, Uswatte G, Taub E, Weiller C. Distributed form of constraint-induced movement therapy improves functional outcome and quality of life after stroke. Arch Phys Med Rehabil 2005;86:204-9.

http://dx.doi.org/10.1016/j.apmr.2004.05.007

27.Taub E, Uswatte G, King DK, Morris D, Crago GE, Chatterjee A. A placebo-controlled trial of constraint-induced movement therapy for upper extremity after stroke. Stroke 2006;37:1045-9.
http://dx.doi.org/10.1161/01.STR.0000206463.66461.97

28.Vaz DV. Terapia de movimento induzido pela restrição na hemiplegia: um estudo de caso único. Fisioter Pesqui 2008;15:298-303.

29. Weingarden $H$, Ring H. Functional electrical stimulation-induced neural changes and recovery after stroke. Euro Medicophys 2006;42:87-90.

30.Ecard L, Silva APS, Neto MP, Veiga H, Cagy M, Piedade R, et al. Os efeitos da estimulação elétrica funcional na assimetria cortical inter-hemisférica. Arqui Neuropsiquiatr 2007;65(3a):642-6.

http://dx.doi.org/10.1590/S0004-282X2007000400019 